\title{
Almost oscillatory three-dimensional dynamical system
}

\author{
Elvan Akin-Bohner ${ }^{1 *}$, Zuzana Došlá ${ }^{2}$ and Bonita Lawrence ${ }^{3}$
}

\footnotetext{
* Correspondence: akine@mst.edu ${ }^{1}$ Missouri University of Science and Technology, 310 Rolla Building, Missouri 65409-0020, USA

Full list of author information is available at the end of the article
}

\section{Abstract}

In this article, we investigate oscillation and asymptotic properties for 3D systems of dynamic equations. We show the role of nonlinearities and we apply our results to the adjoint dynamic systems.

2010 Mathematics Subject Classification: 39A10

Keywords: Time scales, Oscillation, Three-dimensional dynamical system

\section{Introduction}

In this article, we investigate $3 \mathrm{D}$ dynamical systems of the form

$$
\left\{\begin{array}{l}
x^{\Delta}(t)=a(t) f(y(t)) \\
y^{\Delta}(t)=b(t) g(z(t)) \\
z^{\Delta}(t)=\lambda c(t) h(x(t))
\end{array}\right.
$$

on a time scale $\mathbb{T}$, i.e., a closed subset of real numbers, $\lambda= \pm 1, a, b: \mathbb{T} \mapsto[0, \infty)$ (not identically zero) and $c: \mathbb{T} \mapsto[0, \infty)$ are rd-continuous functions such that

$$
\int_{T}^{\infty} a(\tau) \Delta \tau=\int_{T}^{\infty} b(\tau) \Delta \tau=\infty, \quad T \in \mathbb{T}
$$

and $f, g, h: \mathbb{R} \mapsto \mathbb{R}$ are continuous functions such that

$$
u f(u)>0, \quad u g(u)>0, \quad \text { and } u h(u)>0 \text { for } u \neq 0 .
$$

Here we would like to indicate that none of the functions $f, g$ or $h$ are monotonic. Sometimes we will assume that functions $f, g$, and $h$ satisfy

$$
\frac{f(u)}{\Phi_{\alpha}(u)} \geq F, \quad \frac{g(u)}{\Phi_{\beta}(u)} \geq G \frac{h(u)}{\Phi_{\gamma}(u)} \geq H, \quad \text { for all } u \neq 0,
$$

where $F, G, H$ are positive constants and $\Phi_{\alpha}, \Phi_{\beta}$, and $\Phi_{\gamma}$ are odd power functions, i. e.,

$$
\Phi_{p}(u)=|u|^{p} \operatorname{sgn} u(p>0), \quad p \in\{\alpha, \beta, \gamma\} .
$$

Here, we consider only unbounded time scales. The theory of time scales is initiated by Stefan Hilger [1] his PhD dissertation in 1988 in order to unify continuous and discrete analysis. The theory of dynamic equations on time scales helps us not only to 
avoid proving results twice but also to extend them for other time scales such as the set of all integer multiples of a number $h>0$, the set of all integer powers of a number $q>1$. We refer readers the books by Bohner and Peterson [2,3] for an excellent introduction with applications and advances in dynamic equations.

The aim of this article is to study oscillatory and asymptotic properties of solutions of (1). The special case of system (1),

$$
\left\{\begin{array}{l}
x^{\Delta}(t)=a(t) y^{\alpha}(t) \\
y^{\Delta}(t)=b(t) z^{\beta}(t) \\
z^{\Delta}(t)=-c(t) x^{\gamma}(t),
\end{array}\right.
$$

is considered on time scales by Akin-Bohner et al. [4], where $\alpha, \beta, \gamma$ are ratios of odd positive integers. The continuous version of a system similar to system (1) has been considered by Chanturia [5] and the discrete version of system (1) by Schmeidel [6,7] see also references therein. System (4) with $\alpha=\beta=1$ can be written as a third-order difference equation with quasi-differences those oscillatory and asymptotic properties have been investigated in $[8,9]$.

First, we study the case $\lambda=1$. We will obtain asymptotic properties of nonoscillatory solutions of system (1) and we will establish oscillation criteria for system (1). Then we consider the case $\lambda=-1$ and we extend our results proved in [4]. In the last section, we apply our results to adjoint systems and we discuss the role of nonlinearities. Our results are new also for the difference systems.

A solution of system (1) is denoted by $(x, y, z)$. Solution $(x, y, z)$ defined on $\left[t_{0}, \infty\right) \subset \mathbb{T}, t_{0} \in \mathbb{T}$, is said to be proper if

$$
\sup \{|x(s)|,|\gamma(s)|, z(s) \mid: s \in[t, \infty)\}>0 \quad \text { for } t \geq t_{0} .
$$

A proper solution of system (1) is said to be oscillatory if all of its components $x, y, z$ are oscillatory, i.e., neither eventually positive nor eventually negative. Otherwise, proper solution is said to be nonoscillatory. Obviously, if one component of a solution $(x, y, z)$ is eventually of one sign, then all its components are eventually of one sign and so nonoscillatory solutions have all components nonoscillatory.

System (1) with $\lambda=1$ is said to be almost oscillatory, or has Property $B$, if every solution $(x, y, z)$ of system (1) is either oscillatory or

$$
\lim _{t \rightarrow \infty}|x(t)|=\lim _{t \rightarrow \infty}|y(t)|=\lim _{t \rightarrow \infty}|z(t)|=\infty .
$$

System (1) with $\lambda=-1$ is said to be almost oscillatory, or has Property $A$, if every solution $(x, y, z)$ of system (1) is either oscillatory or

$$
\lim _{t \rightarrow \infty}|x(t)|=\lim _{t \rightarrow \infty}|\gamma(t)|=\lim _{t \rightarrow \infty}|z(t)|=0 .
$$

Remark 1.1. The terminology used in the above definitions is not unified in the literature. The terminology Property $A$ and Property $B$ are due to $[5,10]$. As can be noticed in [[11], p. 126] in a picturesque way, Property $A$ and Property $B$ state that every solution which may oscillate, does oscillate. Some authors use a different terminology-the system or higher order equation is almost oscillatory, or strongly oscillatory [11]. 
Changing order of integration is used in our main results. The proof of following lemma can be found in the article by Akin-Bohner and Sun [12].

Lemma 1.1. Let $a, c \in \mathrm{C}_{\mathrm{rd}}\left(\mathbb{T}, \mathbb{R}^{+}\right)$. Then

$$
\int_{T}^{t} c(s)\left(\int_{T}^{\sigma(s)} a(\tau) \Delta \tau\right) \Delta s=\int_{T}^{t} a(s)\left(\int_{s}^{t} c(\tau) \Delta \tau\right) \Delta t .
$$

and

$$
\int_{T}^{t} c(s)\left(\int_{T}^{s} a(\tau) \Delta \tau\right) \Delta s=\int_{T}^{t} a(s)\left(\int_{\sigma(s)}^{t} c(\tau) \Delta \tau\right) \Delta t .
$$

Remark 1.2. Let $a, c \in \mathrm{C}_{\mathrm{rd}}\left(\mathbb{T}, \mathbb{R}^{+}\right)$such that

$$
\int_{T}^{\infty} c(s) \Delta s<\infty
$$

Then

$$
\int_{T}^{\infty} a(t)\left(\int_{t}^{\infty} c(s) \Delta s\right) \Delta t=\int_{T}^{\infty} c(t)\left(\int_{T}^{\sigma(t)} a(s) \Delta s\right) \Delta t .
$$

\section{Nonoscillatory solutions of system (1): case $\lambda=1$}

Throughout this and the following section, we consider the system (1), when $\lambda=1$.

In this section, we study asymptotic properties of nonoscillatory solutions which we use in the following section. The following lemma is the analogy of a lemma in [10].

Lemma 2.1. Assume that $(x, y, z)$ is a nonoscillatory solution of system (1) with $\lambda=1$. For large $t \in \mathbb{T}$, let

$$
\begin{aligned}
& \text { Type }(a): \quad \operatorname{sgn} x(t)=\operatorname{sgn} y(t)=\operatorname{sgn} z(t), \\
& \text { Type }(c): \quad \operatorname{sgn} x(t)=\operatorname{sgn} y(t) \neq \operatorname{sgn} z(t) .
\end{aligned}
$$

Then every nonoscillatory solution of system (1) with $\lambda=1$ is of either Type (a) or Type (c).

Proof. Let $(x, y, z)$ be a nonoscillatory solution of system (1). Without loss of generality, we assume that $x(t)>0$ for $t \geq T, T \in \mathbb{T}$. Then we have $y(t)$ and $z(t)$ are monotone for $t \geq T$. Since $y$ is monotonic, we have either $y(t)<0$ or $y(t)>0$ for all $t \geq T$. Similarly, $z(t)<0$ or $z(t)>0$ for all $t \geq T$.

First, let $z(t)>0$ for $t \geq T$. Suppose $y(t)<0$ for large $t$. Since $z$ is positive and increasing, there exists $\ell>0$ such that $z(t) \geq \ell$ for large $t$. From here and integrating the second equation we get $\gamma(t)-\gamma(T) \geq \ell \int_{T}^{t} b(s) \Delta s$, thus $y(t) \rightarrow \infty$, which is a contradiction with the fact that $y(t)<0$. Therefore, this case is not possible, and so $(x, y, z)$ is of Type (a).

Now let $z(t)<0$ for $t \geq T$. Suppose that $y(t)<0$ for large $t$. It implies that $x$ is eventually positive decreasing. Integrating the first equation, we get $x(t) \rightarrow \infty$, which is a 
contradiction with the boundedness of $x$. Therefore, this case is not possible, and so ( $x$, $y, z)$ is of Type (c).

The proof for the case when $x(t)<0$ for large $t$ is analog.

Solutions of Type (a) are sometimes called strongly monotone solutions (see e.g., [10]).

Lemma 2.2. Any Type (a) solution ( $x, y, z)$ of system (1) with $\lambda=1$ satisfies

$$
\lim _{t \rightarrow \infty}|x(t)|=\infty, \lim _{t \rightarrow \infty}|y(t)|=\infty .
$$

Proof. Let $(x, y, z)$ be a Type (a) solution of system (1) such that all the signs of all three components are eventually positive. Then there exists $T \in \mathbb{T}$ such that $x(t)>0$ and $y(t)>0$ and $z(t)>0$ for $t \geq T$. Using the first equation of system (1) and noting that $y$ is eventually increasing, there exist $T_{1} \geq T, t_{1} \in \mathbb{T}$ and a constant $K>0$ such that $f(y(t)) \geq K$ for $t \geq T_{1}$, we have

$$
x^{\Delta}(t)=a(t) f(\gamma(t)) \geq a(t) K .
$$

Integrating the above inequality from $T_{1}$ to $T_{1} \in \mathbb{T}, T_{1} \geq T$ yields

$$
x(t) \geq x\left(T_{1}\right)+K \int_{T_{1}}^{t} a(\tau) \Delta \tau \geq K \int_{T_{1}}^{t} a(\tau) \Delta \tau .
$$

This implies that $\lim _{t \rightarrow \infty} x(t)=\infty$ by (2).

Using the second equation of system (1) and noting that $z$ is eventually increasing, there exist $T_{3} \geq T_{2}, T_{3} \in \mathbb{T}$ and a constant $L>0$ such that $g(z(t)) \geq L$ for $t \geq T_{3}$ we have

$$
\gamma^{\Delta}(t)=b(t) g(z(t)) \geq b(t) L .
$$

Integrating the above inequality from $T_{3}$ to $t, T_{3} \in \mathbb{T}, T_{3} \geq T_{2}$ gives us

$$
\gamma(t) \geq \gamma\left(T_{3}\right)+L \int_{T_{3}}^{t} b(s) \Delta s \geq L \int_{T_{3}}^{t} b(s) \Delta s .
$$

From the above inequality, (2) implies $\lim _{t \rightarrow \infty} y(t)=\infty$. $\square$

Theorem 2.1. Assume that there exist positive constants $F, H$ and $\alpha, \gamma$ such that

$$
\frac{f(u)}{\Phi_{\alpha}(u)} \geq F, \quad \frac{h(u)}{\Phi_{\gamma}(u)} \geq H \quad \text { for large } u \neq 0,
$$

and

$$
\int_{T}^{\infty} c(\tau)\left(\int_{T}^{\tau} a(s)\left(\int_{T}^{s} b(\tau) \Delta \tau\right)^{\alpha} \Delta s\right)^{\gamma} \Delta \tau=\infty, \quad T \in \mathbb{T} .
$$

Then any Type (a) solution ( $x, y, z)$ of system (1) with $\lambda=1$ satisfies (5).

Proof. Let $(x, y, z)$ be a Type (a) solution of system (1) such that $x(t)>0$ and $y(t)>0$ and $z(t)>0$ for $t \geq T$. By Lemma 2.2 it is sufficient to prove that $\lim _{t \rightarrow \infty}|z(t)|=\infty$. Using the estimation (7), there exists $T_{1} \geq T_{3}, T_{1} \in \mathbb{T}$, such that $f(y(t)) \geq F y^{\alpha}(t)$ for $t \geq$ $T_{1}$. From here and by integration of the first equation of system (1) we get we have 


$$
x(t) \geq F \int_{T_{1}}^{t} a(\tau) y^{\alpha}(\tau) \Delta \tau \geq F L^{\alpha} \int_{T_{1}}^{t} a(\tau)\left(\int_{T_{1}}^{\tau} b(s) \Delta s\right)^{\alpha} \Delta \tau .
$$

Using the third equation of system (1) and the estimation (9), there exist $T_{2} \geq T_{1}, T_{2} \in \mathbb{T}$, and a constant $H>0$ such that $h(x(t)) \geq H x^{\gamma}(t)$ for $t \geq T_{2}$, we have

$$
z^{\Delta}(t)=c(t) h(x(t)) \geq c(t) H x^{\gamma}(t) \geq H F^{\gamma} L^{\alpha \gamma} c(t)\left(\int_{T_{2}}^{t} a(s)\left(\int_{T}^{\tau} b(v) \Delta v\right)^{\alpha} \Delta s\right)^{\gamma} \quad \text { for } t \geq T_{2} .
$$

Integrating this expression from $T_{2}$ to $t$ gives us

$$
z(t) \geq H F^{\gamma} L^{\alpha \gamma} \int_{T_{2}}^{t} c(s)\left(\int_{T 1}^{s} a(\tau)\left(\int_{T}^{\tau} b(v) \Delta v\right)^{\alpha} \Delta \tau\right)^{\gamma} \Delta s .
$$

As $t \rightarrow \infty, \lim _{t \rightarrow \infty} z(t)=\infty$ by (8). By Lemma 2.2, the proof is completed.

We conclude this section with the property Type (c) solution of (1) which will be needed in the following section.

Lemma 2.3. Assume that $(x, y, z)$ is a Type (c) solution of system (1) with $\lambda=1$. Then

$$
\lim _{t \rightarrow \infty} z(t)=0 .
$$

Proof. Assume that $(x, y, z)$ is a nonoscillatory Type (c) solution of system (1). Without loss of generality, assume that $x(t)>0$ for $t \geq T_{1}, T_{1} \in \mathbb{T}$. Then $y(t)>0$ and $z(t)<0$ for $t \geq T_{1}$. Since $z$ is increasing, $\lim _{t \rightarrow \infty} z(t) \leq 0$. Suppose that $\lim _{t \rightarrow \infty} z(t)=l<0$. Then there exists $T_{2} \geq T_{1}, T_{2} \in \mathbb{T}$ such that $g(z(t)) \leq l, t \geq T_{2}$. Integrating the second equation of system (1) from $T_{1}$ to $t$, we have

$$
y(t) \leq y\left(T_{2}\right)+l \int_{T_{2}}^{t} b(\tau) \Delta \tau, \quad t \geq T_{2}
$$

and therefore (2) implies that $\lim _{t \rightarrow \infty} y(t)=-\infty$. But this contradicts the fact that $y(t)$ $>0$ for $t \geq T_{1}$. Therefore, $\lim _{t \rightarrow \infty} z(t)=0$.

\section{Almost oscillatory system: case $\lambda=1$}

We now establish conditions that system (1) is almost oscillatory.

Observe that by Lemmas 2.1 and 2.2, every bounded solution is either Type (c) or oscillatory. Hence, if system (1) is almost oscillatory, then every bounded solution is oscillatory.

Our first result is

Theorem 3.1. Assume

$$
\int_{T}^{\infty} c(s) \Delta s=\infty, \quad T \in \mathbb{T} .
$$

Then system (1) with $\lambda=1$ is almost oscillatory. 
Proof. From Lemma 2.1, we know that nonoscillatory solutions are either Type (a) or Type (c). Assume $(x, y, z)$ is a Type (c) solution of system (1). Without loss of generality, assume that there exists $T \in \mathbb{T}$ such that $x(t)>0, y(t)>0$, and $z(t)<0$ for $t \geq T$. Since $x$ is eventually increasing, there exists $l>0$ such that $h(x(t)) \geq l$ for $t \geq T$. Integrating the third equation of system (1) and using the positivity and the monotonicity of $x$ we have

$$
z(t) \geq z(T)+l \int_{T}^{t} c(\tau) \Delta \tau, \quad t \geq T
$$

and so (10) implies $\lim _{t \rightarrow \infty} z(t)=\infty$. This contradicts the assumptions on $z$. Therefore, solutions of Type (c) are not possible. If $(x, y, z)$ is a Type (a), then from Lemma 2.2 and (10), we obtain (5). $\square$

Remark 3.1. Theorem 3.1 extends [[6], Theorem 2] for difference systems where it is proved that every bounded solution of (1) with $\lambda=1$ is oscillatory.

Theorem 3.2. Let there exists $G>0$ and $\beta \leq 1$ such that

$$
\frac{g(u)}{\Phi_{\beta}(u)} \geq G \quad \text { for all } u \neq 0,
$$

and

$$
\int_{T}^{\infty} c(s) \int_{T}^{\sigma(s)} b(\tau) \Delta \tau \Delta s=\infty .
$$

Then every nonoscillatory solution of (1) with $\lambda=1$ is a strongly monotone solution.

In addition, if for some $F>0$ and $\alpha>0$

$$
\frac{f(u)}{\Phi_{\alpha}(u)} \geq F \quad \text { for all } u \neq 0,
$$

and (8) holds, then system (1) with $\lambda=1$ is almost oscillatory.

Proof. Assume $(x, y, z)$ is a Type (c) solution of system (1). Without loss of generality, assume that there exists $T \in \mathbb{T}$ such that $x(t)>0, y(t)>0$ and $z(t)<0$ for $t \geq T$. Since $x$ is eventually increasing, there exists $l>0$ such that $h(x(t)) \leq l$ for large $t$. By Lemma 2.3 we have $\lim _{t \rightarrow \infty} z(t)=0$ and so

$$
-z(t)=\int_{t}^{\infty} c(s) h(x(s)) \Delta s \geq \ell \int_{t}^{\infty} c(s) \Delta s .
$$

Using (11) and the fact $\beta \leq 1$, we have for large $t$

$$
g(-z(t)) \geq G(-z(t))^{\beta} \geq G(-z(t)) \geq G \ell \int_{t}^{\infty} c(s) \Delta s .
$$

Integrating the second equation of system (1) we have

$$
\gamma(t)-\gamma(T)=\int_{T}^{t} b(s) g(z(s)) \Delta s,
$$


thus

$$
-\gamma(t)+\gamma(T)=\int_{T}^{t} b(s) g(-z(s)) \Delta s \geq G \ell \int_{T}^{t} b(s) \int_{T}^{t} c(\tau) \Delta \tau \Delta s .
$$

Using Lemma 1.1 in the last integration we get

$$
-\gamma(t)+\gamma(T) \geq G \ell \int_{T}^{t} c(s) \int_{T}^{\sigma(s)} b(\tau) \Delta \tau \Delta s .
$$

Passing $t \rightarrow \infty$ and using (12), we get a contradiction with the boundedness of $y$.

The second statement follows from Theorem 2.1.

Theorem 3.3. Assume (3) and $\alpha \beta \gamma>1$. If

$$
\int_{T}^{\infty} a(t)\left(\int_{\sigma(t)}^{\infty} b(s)\left(\int_{s}^{\infty} c(\tau) \Delta \tau\right)^{\beta} \Delta s\right)^{\alpha} \Delta t=\infty,
$$

then every nonoscillatory solution of (1) with $\lambda=1$ is a strongly monotone solution. In addition, if (8) holds, then system (1) with $\lambda=1$ is almost oscillatory.

Proof. By Lemma 2.1 any nonoscillatory solution of system (1) is either of Type (a) or (c) for $t \geq T, T \in \mathbb{T}$. We show that a nonoscillatory solution of system (1) of Type (c) cannot occur. Assume that there exists a nonoscillatory solution $(x, y, z)$ of system (1) of Type (c) for $t \geq T$. Without loss of generality, we assume that $x(t)>0$ for $t \geq T$.

By Lemma 2.3 we have $\lim _{t \rightarrow \infty} z(t)=0$. Since $y$ is eventually decreasing, there exists $\lim _{t \rightarrow \infty} \gamma(t)=l \geq 0$. Integrating third and second equations, we obtain

$$
-z(t) \geq \int_{t}^{\infty} c(\tau) x^{\gamma}(\tau) \Delta \tau
$$

and

$$
\gamma(\sigma(t))=l-\int_{\sigma(\tau)}^{\infty} b(\tau) z^{\beta}(\tau) \Delta \tau .
$$

Since $y$ is eventually decreasing, $l \geq 0$ and $x$ is eventually increasing, by (14) we have

$$
\begin{aligned}
y(t) \geq \gamma(\sigma(t)) & =l+\int_{\sigma(\tau)}^{\infty} b(\tau)\left(\int_{\tau}^{\infty} c(s) x^{\gamma}(s) \Delta s\right)^{\beta} \Delta \tau \\
& \geq \int_{\sigma(\tau)}^{\infty} b(\tau) x^{\gamma \beta}(\tau)\left(\int_{\tau}^{\infty} c(s) \Delta s\right)^{\beta} \Delta \tau \\
& \geq x^{\gamma \beta}(\sigma(t)) \int_{\sigma(\tau)}^{\infty} b(\tau)\left(\int_{\tau}^{\infty} c(s) \Delta s\right)^{\beta} \Delta \tau .
\end{aligned}
$$


This implies that

$$
\gamma^{\alpha}(t) \geq x^{\alpha \gamma \beta}(\sigma(t))\left(\int_{\sigma(t)}^{\infty} b(\tau)\left(\int_{\tau}^{\infty} c(s) \Delta s\right)^{\beta} \Delta \tau\right)^{\alpha} .
$$

Multiplying this inequality by $a$, dividing it by $x^{\alpha \beta \gamma}(\sigma(t))$, and using the first equation gives us

$$
\frac{x^{\Delta}(t)}{x^{\alpha \beta \gamma}(\sigma(t))} \geq a(t)\left(\int_{\sigma(t)}^{\infty} b(\tau)\left(\int_{\tau}^{\infty} c(s) \Delta s\right)^{\beta} \Delta \tau\right)^{\alpha}
$$

and so

$$
\int_{T_{1}}^{t} \frac{x^{\Delta}(\tau)}{x^{\alpha \beta \gamma}(\sigma(\tau))} \Delta \tau \geq \int_{T_{1}}^{t} a(\tau)\left(\int_{\sigma(t)}^{\infty} b(s)\left(\int_{s}^{\infty} c(v) \Delta v\right)^{\beta} \Delta s\right)^{\alpha} \Delta \tau .
$$

On the other hand, by the chain rule, [[2], Theorem 1.90] we have

$$
\left(\frac{1}{x^{\alpha \beta \gamma-1}(t)}\right)^{\Delta}=(1-\alpha \beta \gamma) x^{\Delta}(t) \int_{0}^{1} \frac{1}{\left(x(t)+\mu(t) h x^{\Delta}(t)\right)^{\alpha \beta \gamma}} d h .
$$

Since $0<x(t) \leq x(t)+\mu h x^{\Delta}(t) \leq x(t)+\mu x^{\Delta}(t)=x^{\sigma}(t)$,

$$
\begin{aligned}
& \left(\frac{1}{x^{\alpha \beta \gamma-1}(t)}\right)^{\Delta} \leq(1-\alpha \beta \gamma) \frac{x^{\Delta}(t)}{x^{\alpha \beta \gamma}(\sigma(t))} \\
& \frac{1}{1-\alpha \beta \gamma}\left(\frac{1}{x^{\alpha \beta \gamma-1}(t)}\right)^{\Delta} \geq \frac{x^{\Delta}(t)}{x^{\alpha \beta \gamma}(\sigma(t))}
\end{aligned}
$$

Integrating the above inequality from $T_{1}$ to $t$ yields

$$
\frac{1}{1-\alpha \beta \gamma}\left[x^{1-\alpha \beta \gamma}(t)-x^{1-\alpha \beta \gamma}\left(T_{1}\right)\right] \geq \int_{T_{1}}^{t} \frac{x^{\Delta}(\tau)}{x^{\alpha \beta \gamma}(\sigma(\tau))} \Delta \tau
$$

As $t \rightarrow \infty$, we obtain that

$$
\int_{T_{1}}^{\infty} \frac{x^{\Delta}(\tau)}{x^{\alpha \beta \gamma}(\sigma(\tau))} \Delta \tau<\infty
$$

but this contradicts (15) as $t \rightarrow \infty$. $\square$

Remark 3.2. Theorem 3.3 extends [[5], Theorem 2.4] stated for differential systems.

\section{Almost oscillatory system: case $\lambda=-1$}

In this section, we study the case $\lambda=-1$.

We start with the classification of solutions of system (1) with $\lambda=-1$. This is an analogue of Kiguradze lemma.

Lemma 4.1. Assume that $(x, y, z)$ is a nonoscillatory solution of system (1) with $\lambda=$ -1 . For large $t \in \mathbb{T}$, let 
Type (a): $\operatorname{sgn} x(t)=\operatorname{sgn} y(t)=\operatorname{sgn} z(t)$,

Type (b) : $\quad \operatorname{sgn} x(t)=\operatorname{sgn} z(t) \neq \operatorname{sgn} y(t)$.

Then every nonoscillatory solution of system (1) with $\lambda=-1$ is either Type (a) or Type (b).

Proof. It is similar as the proof [[4], Lemma 3.2], so the details are omitted.

Solution of Type (b) are sometimes called Kneser solutions (see e.g., [10]).

Lemma 4.2. Assume $(x, y, z)$ is a Type $(b)$ solution of system (1) with $\lambda=-1$. Then

$$
\lim _{t \rightarrow \infty} \gamma(t)=\lim _{t \rightarrow \infty} z(t)=0 .
$$

Proof. Assume $(x, y, z)$ is a Type (b) solution of system (1) and $x(t)>0, y(t)<0$, and $z(t)>0$ for $t \geq T_{1}\left(T_{1} \in \mathbb{T}\right)$. Since $y(t)$ is increasing, we have $\lim _{t \rightarrow \infty} y(t) \leq 0$. Assume $\lim$ $\lim _{t \rightarrow \infty} y(t) \neq 0$. Then there exist $T_{2} \geq T_{1}$ and a constant $l<0$ such that $f(y(t)) \leq l$ for $t$ $\geq T_{2}$. Integrating the first equation of system (1) from $T_{2}$ to $t$, we have

$$
x(t) \leq x\left(T_{2}\right)+l \int_{T_{2}}^{t} a(\tau) \Delta \tau, \quad t \geq T_{2}
$$

and so (2) implies $\lim _{t \rightarrow \infty} x(t)=-\infty$. This contradicts our assumptions about the nature of $x$ and therefore $\lim _{t \rightarrow \infty} y(t)=0$. In a similar way, we can show that $\lim _{t \rightarrow \infty} z(t)=0$.

Theorem 4.1. Assume (10). Then system (1) with $\lambda=-1$ is almost oscillatory.

Proof. Assume $(x, y, z)$ is a nonoscillatory solution of system (1). By Lemma 4.1, nonoscillatory solutions are either Type (a) or Type (c). Assume $(x, y, z)$ is a Type (a) solution. Without loss of generality, assume that there exists $T \in \mathbb{T}$ such that $x(t)>0, y(t)$ $>0$, and $z(t)>0$ for $t \geq T$. Since $x$ is eventually increasing, there exists $l>0$ such that $h(x(t)) \geq l$ for $t \geq T$. Integrating the third equation of system (1) and using the positivity and the monotonicity of $x$ we have

$$
z(t) \geq z(T)+l \int_{T}^{t} c(\tau) \Delta \tau, \quad t \geq T
$$

and so (10) implies $\lim _{t \rightarrow \infty} z(t)=\infty$ which is a contradiction with the boundedness of $z$. Therefore, solutions of Type (a) are not possible. If $(x, y, z)$ is a Type (c), then in view

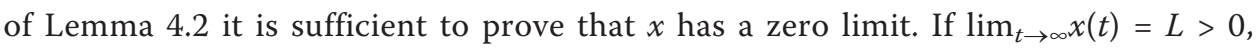
then integrating the third equation from $T$ to $t$ we have

$$
z(t) \leq z(T)-L \int_{T}^{t} c(\tau) \Delta \tau
$$

and passing $t \rightarrow \infty$ we get a contradiction with the boundedness of $z$. $\square$

Next we assume

$$
\int_{T}^{\infty} c(t) \Delta t<\infty, \quad T \in \mathbb{T} .
$$

Similarly, as in [[4], Theorem 3.1] for system (4) the following holds. 
Theorem 4.2. Assume that there exist positive constants $F, G$ and $\alpha, \beta$ such that

$$
\frac{f(u)}{\Phi_{\alpha}(u)} \geq F, \quad \frac{g(u)}{\Phi_{\beta}(u)} \geq G \quad \text { for small } u \neq 0 .
$$

If either

$$
\int_{T}^{\infty} b(t)\left(\int_{t}^{\infty} c(s) \Delta s\right)^{\beta} \Delta t=\infty
$$

or

$$
\int_{T}^{\infty} a(t)\left(\int_{t}^{\infty} b(s)\left(\int_{s}^{\infty} c(\tau) \Delta \tau\right)^{\beta} \Delta s\right)^{\alpha} \Delta t=\infty
$$

then every Kneser solution of system (1) with $\lambda=-1$ satisfies $\lim _{t \rightarrow \infty} x(t)=0$.

Proof. Without loss of generality, assume that $(x, y, z)$ is a Kneser solution of system (1) such that $x(t)>0$ for $t \geq t_{0}, t_{0} \in[0, \infty)$. From the first equation of system (1), $x$ is nonincreasing, and $\lim _{t \rightarrow \infty} x(t)=L_{1}<\infty$. By Lemma 4.2, $\lim _{t \rightarrow \infty} y(t)=\lim _{t \rightarrow \infty} z(t)=0$. We now show that $\lim _{t \rightarrow \infty} x(t)=0$, and so we let $\lim _{t \rightarrow \infty} x(t)=L_{1}>0$. Then there exists $T_{1} \geq$ $t_{0}, T_{1} \in[0, \infty)$ and $L>0$ such that $h(x(t))>L^{\beta}$ for $t \geq T_{1}$.

Integrating the third equation from $t$ to $\infty$ we have

$$
z(t) \geq L \int_{t}^{\infty} c(s) \Delta s
$$

and so

$$
g(z(t)) \geq G z^{\beta}(t) \geq G L^{\beta}\left(\int_{t}^{\infty} c(\tau) \Delta \tau\right)^{\beta} .
$$

Hence, integrating the second equation of system (1) from $T$ to $t$ we have

$$
y(t)-\gamma(T) \geq \int_{T}^{t} b(\tau) g(z(\tau)) \Delta \tau \geq G L^{\beta} \int_{T}^{t} b(\tau)\left(\int_{\tau}^{\infty} c(s) \Delta s\right)^{\beta} \Delta \tau .
$$

If (17), then $\lim _{t \rightarrow \infty} \gamma(t)=-\infty$, which is a contradiction.

Assume (18). Integrating the second equation of system (1) from $t$ to $\infty$ and using the fact $\lim _{t \rightarrow \infty} y(t)=0$, we get the estimation

$$
-\gamma(t) \geq \int_{t}^{\infty} b(\tau) g(z(\tau)) \Delta \tau \geq G L^{\beta} \int_{t}^{\infty} b(\tau)\left(\int_{\tau}^{\infty} c(s) \Delta s\right)^{\beta} \Delta \tau .
$$


Therefore integrating the first equation of (1) from $T_{1}$ to $t$

$$
-x(t)+x\left(T_{1}\right) \geq F \int_{T_{1}}^{t} a(s)(-\gamma)^{\alpha}(s) \Delta s,
$$

we have

$$
-x(t)+x\left(T_{1}\right) \geq G^{\alpha} F L_{1}^{\alpha \beta \gamma} \int_{T_{1}}^{t} a(s)\left(\int_{s}^{\infty} b(\tau)\left(\int_{\tau}^{\infty} c(u) \Delta u\right)^{\beta} \Delta \tau\right)^{\alpha} \Delta s .
$$

This implies that $\lim _{t \rightarrow \infty} x(t)=-\infty$, a contradiction. $\square$

Theorem 4.3. Assume (3) and $\alpha \beta \gamma<1$. If

$$
\int_{T_{2}}^{\infty} c(\tau)\left(\int_{T_{2}}^{\tau} a(s)\left(\int_{T_{1}}^{s} b(v) \Delta v\right)^{\alpha} \Delta s\right)^{\gamma} \Delta \tau=\infty
$$

then every nonoscillatory solution of (1) with $\lambda=-1$ is a Kneser solution.

In addition, if (17) holds, then system (1) with $\lambda=-1$ is almost oscillatory.

Proof. Suppose $(x, y, z)$ is a nonoscillatory solution of system (1). By Lemma 4.1, each nonoscillatory solution of system (1) is either Type (a) or Type (b). Without loss of generality, we assume that $x(t)>0$ for $t \geq T_{1}, T_{1} \in \mathbb{T}$.

Assume $(x, y, z)$ is of Type (a). Integrating the second equation of system (1) from $T_{1}$ to $t$ and using the positivity of $y$ yields

$$
y(t)>\int_{T_{1}}^{t} b(\tau) g(z(\tau)) \Delta \tau, \quad t \geq T_{1} .
$$

Since $z(t)$ is positive decreasing for $t \geq T_{1}$ from (3) we have

$$
\gamma(t) \geq \mathrm{G} z^{\beta}(t) \int_{T_{1}}^{t} b(\tau) \Delta \tau
$$

Integrating the first equation of system (1) from $T_{2} \in \mathbb{T}\left(T_{2} \geq T_{1}\right)$ to $t$ and using (3) gives us

$$
x(t) \geq F \int_{T_{2}}^{t} a(\tau) \gamma^{\alpha}(\tau) \Delta \tau .
$$

From (20) and the monotonicity of $z$ we conclude that

$$
x(t) \geq F G^{\alpha}(z(t))^{\alpha \beta} \int_{T_{2}}^{t} a(\tau)\left(\int_{T_{1}}^{\tau} b(s) \Delta s\right)^{\alpha} \Delta \tau, \quad T_{2} \geq T_{1} .
$$


Using the preceding inequality and (3), the third equation of system (1) yields

$$
\begin{aligned}
-z^{\Delta}(t) & =c(t) h(x(t)) \geq H c(t) x^{\gamma}(t) \\
& \geq c(t)\left(F G^{\alpha} z^{\alpha \beta}(t) \int_{T_{2}}^{t} a(\tau)\left(\int_{T_{1}}^{\tau} b(s) \Delta s\right)^{\alpha} \Delta \tau\right)^{\gamma} \\
& =c(t)\left(F G^{\alpha}\right)^{\gamma} z^{\alpha \beta \gamma}\left(\int_{T_{2}}^{t} a(\tau)\left(\int_{T_{1}}^{\tau} b(s) \Delta s\right)^{\alpha} \Delta \tau\right)^{\gamma}
\end{aligned}
$$

Dividing the above inequality by $z^{a \beta \gamma}(t)$ and integrating it from $T_{2}$ to $t$ yields

$$
\int_{T_{2}}^{t}-\frac{z^{\Delta}(\tau)}{z^{\alpha \beta \gamma}(\tau)} \Delta \tau \geq\left(F G^{\alpha}\right)^{\gamma} \int_{T_{2}}^{t} c(\tau)\left(\int_{T_{2}}^{\tau} a(s)\left(\int_{T_{1}}^{s} b(v) \Delta v\right)^{\alpha} \Delta s\right)^{\gamma} \Delta \tau .
$$

Now we prove that

$$
\int_{T}^{\infty} \frac{-z^{\Delta}(\tau)}{z^{\alpha \beta \gamma}(t)} \Delta \tau<\infty \quad T \in[0, \infty) .
$$

We proceed by the similar argument as in the proof of Theorem 3.3. We have

$$
\left(\frac{1}{z^{\alpha \beta \gamma-1}(t)}\right)^{\Delta}=(-\alpha \beta \gamma+1) z^{\Delta}(t) \int_{0}^{1} \frac{1}{\left(z(t)+\mu(t) h z^{\Delta}(t)\right)^{\alpha \beta \gamma}} d h,
$$

thus, because $z(t) \geq z(t)+\mu(t) h z^{\Delta}(t) \geq z^{\sigma}(t)>0$, and $z$ is positive decreasing,

$$
-\frac{z^{\Delta}(t)}{z^{\alpha \beta \gamma}(t)} \leq \frac{1}{\alpha \beta \gamma-1}\left(\frac{1}{z^{\alpha \beta \gamma-1}(t)}\right)^{\Delta} .
$$

Integrating above inequality from $T$ to $t$ we obtain

$$
\begin{aligned}
\int_{T}^{t} \frac{-z^{\Delta}(\tau)}{z^{\alpha \beta \gamma}(\tau)} \Delta \tau & \leq \frac{1}{\alpha \beta \gamma-1} \int_{T}^{t}\left(\frac{1}{z^{\alpha \beta \gamma-1}(\tau)}\right)^{\Delta} \Delta \tau \\
& =\frac{1}{\alpha \beta \gamma-1}\left[\frac{1}{z^{\alpha \beta \gamma-1}(t)}-\frac{1}{z^{\alpha \beta \gamma-1}\left(T_{1}\right)}\right],
\end{aligned}
$$

which yields (21). Hence, passing $t \rightarrow \infty$ in (4), we get a contradiction. Therefore, system (1) cannot have Type (a) solutions. So, every nonoscillatory solution is of Type (b). By Lemma 4.2 and Theorem 4.2, we have (6). So this completes the proof.

Remark 4.1. Theorem 4.3 extends [[6], Theorem 1] stated for difference systems.

\section{Conclusion and application to adjoint systems}

In this section, we consider (4) where $\lambda= \pm 1$ and we summarize the above results.

We conclude that if (10) holds, then, independently on the nonlinearities, (1) is almost oscillatory for $\lambda= \pm 1$. If (16) holds, then, in general, almost oscillation depends on the sign of $\lambda$ and on the types of nonlinearities. 
Consider the following adjoint systems:

$$
\left\{\begin{array}{l}
x^{\Delta}(t)=a(t) y(t) \\
y^{\Delta}(t)=b(t) z(t) \\
z^{\Delta}(t)=c(t)(x(t))^{\gamma}
\end{array}\right.
$$

and

$$
\left\{\begin{array}{l}
x^{\Delta}(t)=b(t) y(t) \\
y^{\Delta}(t)=a(t) z(t) \\
z^{\Delta}(t)=-c(t)(x(t))^{1 / \gamma}
\end{array}\right.
$$

The terminology "adjoint system" is used due to the fact that the corresponding matrices of systems (22) and (23) are adjoint.

Applying our results from Sections 3 and 4 we get conditions when both systems are almost oscillatory.

Corollary 5.1. Let $\gamma=1$. If (12) holds and

$$
\int_{T}^{\infty} c(t)\left(\int_{T}^{\sigma(t)} a(s)\left(\int_{T}^{\sigma(s)} b(\tau) \Delta \tau\right) \Delta s\right) \Delta t=\infty
$$

then linear adjoint systems (22) and (23) are almost oscillatory.

Proof. By Theorem 3.2 we have that (22) is almost oscillatory. Applying two times the change of integration for triple integrals we have

$$
\int_{T}^{\infty} a(t)\left(\int_{t}^{\infty} b(s)\left(\int_{s}^{\infty} c(\tau) \Delta \tau\right) \Delta s\right) \Delta t=\int_{T}^{\infty} c(t)\left(\int_{T}^{\sigma(t)} b(s)\left(\int_{T}^{\sigma(s)} a(\tau) \Delta \tau\right) \Delta s\right) \Delta t .
$$

Thus, interchanging $a$ and $b$, we get from Theorem 4.1 that every Kneser solution of (23) satisfies (6). This together with [[4], Theorem 4.4] result that (23) is almost oscillatory.

Corollary 5.2. Assume that $\gamma>1$,

$$
\int_{T}^{\infty} c(t)\left(\int_{T}^{t} b(s) \Delta s\right)^{1 / \gamma} \Delta t=\infty
$$

and

$$
\int_{T}^{\infty} c(t)\left(\int_{T}^{\sigma(t)} a(s) \Delta s\right) \Delta t=\infty .
$$

Then both systems (22) and (23) are almost oscillatory.

Proof. It follows from Theorems 3.2 and 4.3. $\square$

Acknowledgements

The second author is supported by Grant P201/11/0768 of the Grant Agency of the Czech Republic.

Author details

${ }^{1}$ Missouri University of Science and Technology, 310 Rolla Building, Missouri 65409-0020, USA ${ }^{2}$ Masaryk University Brno, CZ-66295 Brno, Czech Republic ${ }^{3}$ Marshall University Huntington, Huntington, WV 25755, USA 
Authors' contributions

All authors contributed equally to the manuscript and read and approved the final draft.

\section{Competing interests}

The authors declare that they have no competing interests.

Received: 5 March 2012 Accepted: 17 April 2012 Published: 17 April 2012

\section{References}

1. Hilger, S: Ein Maßkettenkalkül mit Anwendung auf Zentrumsmannigfaltigkeiten. PhD thesis Universität Würzburg. (1988)

2. Bohner, M, Peterson, A: Dynamic Equations on Time Scales: An Introduction with Applications. Birkhäuser, Boston (2001)

3. Bohner, M, Peterson, A: Advanced in Dynamic Equations on Time Scales. Birkhäuser, Boston (2003)

4. Akin-Bohner, E, Došlá, Z, Lawrence, B: Oscillatory properties for three-dimensional dynamic systems. Nonlinear Anal. 69, 483-494 (2008)

5. Chanturia, TA: On oscillatory properties of systems of nonlinear ordinary differential equations. Proc I. N. Vekua Inst Appl Math., Tbilisi (in Russian). 14, 163-204 (1983)

6. Schmeidel, E: Oscillation of nonlinear three-dimensional difference systems. Math Bo-hem. 135, 163-170 (2010)

7. Schmeidel, E: Boundedness of solutions of nonlinear three-dimensional difference systems with delays. Fasc Math. 44, 109-113 (2010)

8. Došlá, Z, Kobza, A: Nonoscillatory solutions of third-order difference equations. In: Proc Eighth International Conf Difference Equ Appl. pp. 105-112.CRC, Boca Raton (2005). Chapman \& Hall/CRC, Boca Raton

9. Došlá, Z, Kobza, A: On third-order linear equations involving quasi-differences. Adv Diff Equ. 2006, 1-13 (2006)

10. Kiguradze, IT, Chanturia, TA: Asymptotic Properties of Solutions of Nonautonomous Ordinary Differential Equations. Kluwer Academic Publishers, Dordrecht-Boston-London (1993)

11. Elias, U: Oscillation Theory of Two-Term Differential Equations. Kluwer Academic Publishers, Dordrecht-Boston-London (1997)

12. Akin-Bohner, E, Sun, S: Oscillation criteria for a class of second-order dynamic inclusions. submitted

doi:10.1186/1687-1847-2012-46

Cite this article as: Akin-Bohner et al:: Almost oscillatory three-dimensional dynamical system. Advances in Difference Equations 2012 2012:46.

\section{Submit your manuscript to a SpringerOpen ${ }^{\circ}$} journal and benefit from:

Convenient online submission

- Rigorous peer review

- Immediate publication on acceptance

- Open access: articles freely available online

- High visibility within the field

- Retaining the copyright to your article

Submit your next manuscript at $\boldsymbol{\wedge}$ springeropen.com 